\title{
Coronary Artery Bypass Grafting in an Infant after an Arterial Switch Operation
}

\author{
Wooseok Choi, M.D.', Wonkyung Pyo, M.D. ', Eun Seok Choi, M.D. ${ }^{1,2}$, Cheol Hyun Chung, M.D., Ph.D.' \\ 'Department of Thoracic and Cardiovascular Surgery and 'Division of Pediatric Cardiac Surgery, Department of Thoracic and Cardiovascular Surgery, Asan \\ Medical Center, University of UIsan College of Medicine, Seoul, Korea
}

\section{ARTICLE INFO}

Received June 26, 2020

Revised August 1, 2020

Accepted August 3, 2020

Corresponding author

Cheol Hyun Chung

Tel 82-2-3010-3946

Fax 82-2-3010-6966

E-mail hyun227@amc.seoul.kr

ORCID

https://orcid.org/0000-0001-8981-6011
Coronary artery bypass grafting (CABG) is rarely performed in infants because of its technical difficulty and unclear long-term results. A 90-day-old male infant weighing $3.5 \mathrm{~kg}$ who underwent an arterial switch operation (ASO) for transposition of the great arteries developed left coronary artery insufficiency despite augmentation and reimplantation of the left coronary button. On-pump beating heart CABG was performed using an internal mammary artery graft to revascularize the left anterior descending artery. Postoperative computed tomography angiography revealed that the graft was patent. At 7 months postoperatively, the patient weighed $8.5 \mathrm{~kg}$, and echocardiography revealed good ventricular function. CABG can be an alternative treatment for post-ASO coronary complications in early infancy.

Keywords: Transposition of the great vessels, Coronary artery bypass, Infant

\section{Case report}

A male neonate was born by spontaneous vaginal delivery at 38 weeks of gestation, with a birth weight of $3,110 \mathrm{~g}$. Echocardiography and computed tomography (CT) findings confirmed the antenatal diagnosis of transposition of the great arteries with an intact ventricular septum. CT scan showed inverted origin of the coronary arteries (1R;2LCx). At the age of 10 days, the patient underwent an arterial switch operation (ASO) with the LeCompte maneuver and coronary transfer with the trap-door technique. The patient recovered quickly and was transferred to the general ward on postoperative day 11. Echocardiography showed a left ventricular ejection fraction (LVEF) of $72 \%$. However, on postoperative day 18 , the patient had a sudden onset of diaphoresis and hypotension. Echocardiography revealed severe left ventricular dysfunction with decreased motion of the left ventricular free wall, an estimated ejection fraction of only $25 \%$, and moderate mitral regurgitation due to coaptation failure. CT angiography revealed severe narrowing of the orifice of the left coronary artery (LCA).

At the age of 38 days (postoperative day 28), the patient underwent surgical angioplasty for the LCA button. The LCA button, which was enlarged using an autologous pericardial patch, was reimplanted posteriorly from its previous location. Postoperative coronary angiography showed intact flow in the LCA (Fig. 1A). Echocardiography revealed an improved LVEF of 54\%. However, on the 40th day after the second operation, the patient showed lactic acidosis and ST-segment depression on electrocardiography in the precordial leads, which was aggravated with feeding. Echocardiography revealed a decreased LVEF of 48\%. A follow-up catheterization study showed diffuse narrowing in the left main coronary artery, left anterior descending artery (LAD), and left circumflex artery (LCx) without narrowing of the LCA ostium (Fig. 1B).

At the age of 90 days, the patient's weight was $3.5 \mathrm{~kg}$ and coronary artery bypass grafting (CABG) was planned to revascularize the left coronary arteries. Preoperative aortography showed that the left internal mammary artery (LIMA) had a diameter of $1 \mathrm{~mm}$ (Fig. 2). The operation was performed by a surgeon who was highly experienced in CABG in adults. In the operative field, the LIMA showed good pulsation and seemed prominent, which was attributable to the multiple collaterals to the lungs. The anastomo- 

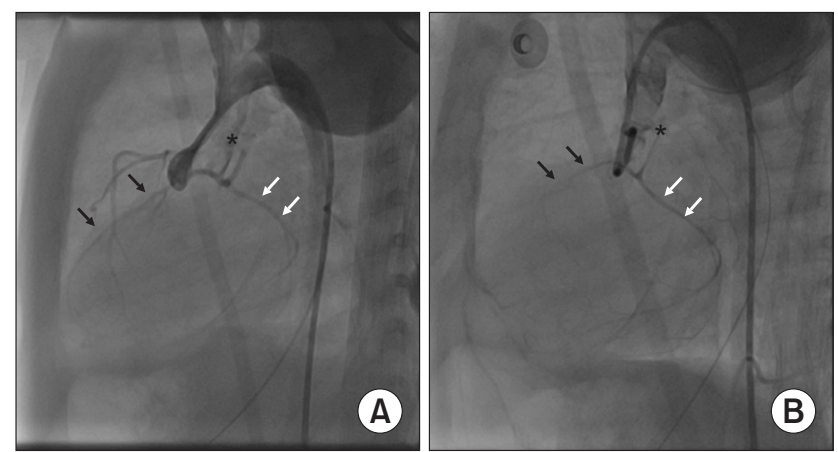

Fig. 1. Coronary angiograms. (A) Coronary angiogram after an operation for left coronary artery reimplantation showing intact flow in the left anterior descending artery (black arrow), left circumflex artery (white arrow), and left coronary os (asterisk). (B) Follow-up angiogram demonstrating diminished flow in the left anterior descending artery (black arrow), left circumflex artery (white arrow), and left coronary os (asterisk).

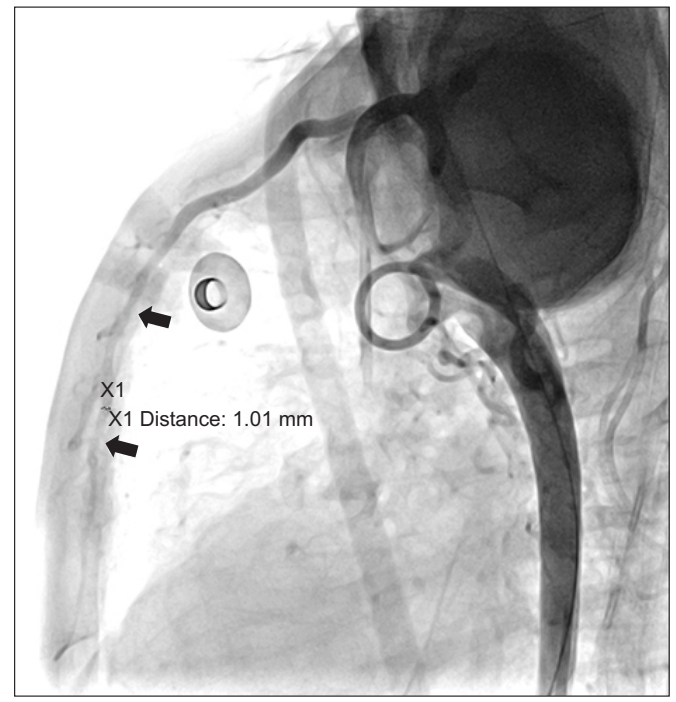

Fig. 2. Preoperative aortogram showing an intact left internal mammary artery (black arrow).

sis site of the LAD could be identified without difficulty. As the LIMA was injured during dissection, a free right internal mammary artery (IMA) graft was used to elongate the bypass graft in a sliding fashion with 8-0 Prolene interrupted sutures. Grafting to the LAD anastomosis was performed using continuous running 8-0 Prolene sutures under an on-pump beating heart using a 1-mm shunt to reduce myocardial ischemia and secure the operative field. The LAD showed good backflow. Transit time flow measurements showed good flow to the LAD $(18 \mathrm{~mL} / \mathrm{min}$, pulsatility index $<2$ ). The patient was successfully weaned from cardiopulmonary bypass after 81 minutes. Postoperative CT showed patent flow from the LIMA to the LAD

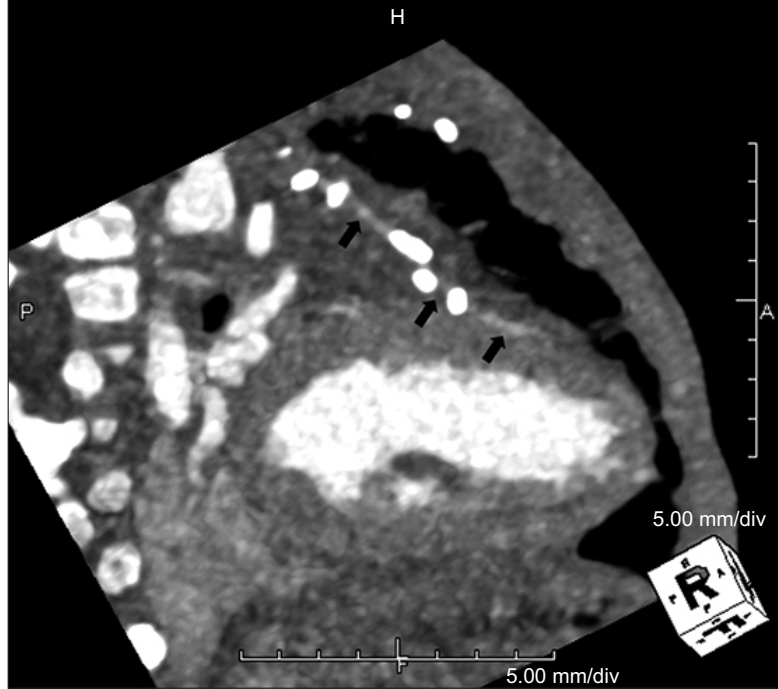

Fig. 3. Postoperative coronary computed tomographic angiogram showing the patency of the left internal mammary artery to the left anterior descending artery graft flow (black arrow).

(Fig. 3), and transthoracic echocardiography revealed good ventricular function, with an ejection fraction of $66.3 \%$. The patient was discharged successfully on postoperative day 14 . Dual antiplatelet therapy with aspirin and clopidogrel was initiated. At the 7-month follow-up, the patient weighed $8.5 \mathrm{~kg}$. Echocardiography obtained at that time revealed good ventricular function with a LVEF of $56.4 \%$ and trivial mitral regurgitation.

This study was approved by the Institutional Review Board of Asan Medical Center (IRB approval no., 20200920). All authors declare that verbal informed consent was obtained from the patient's caregiver for publication of this case report and accompanying images.

\section{Discussion}

In this case, CABG with an IMA graft was used in an infant with a recurrent post-ASO coronary complication. Coronary artery stenosis or obstruction can present as an early or late complication of ASO. It leads to critical myocardial ischemia, increasing early mortality and morbidity. If there is evidence of myocardial ischemia, interventions must be initiated promptly to manage the condition. CABG is a rare procedure in the pediatric population, but it can be performed in selected circumstances.

Surgical angioplasty of the coronary artery is the preferred technique for obstruction after coronary transfer during ASO at many centers [1]. Surgical angioplasty can restore the physiological perfusion of the coronary artery 
and is therefore thought to be more useful than a bypass graft. In this patient, however, angioplasty and reimplantation of the coronary ostium were attempted once, and angiography after the revision revealed an intact coronary ostium without kinking or extrinsic compression (Fig. 1B). This lesion is considered unsuitable for repeated ostial angioplasty. Thus, the decision was made to perform CABG in this case.

The most common indication of CABG in patients with ASO is an intramural course of the coronary artery with difficulties for coronary transfer. In this scenario, urgent IMA grafting is considered because of failure to wean the patient from bypass during the operation. However, in most cases, CABG remains a second option because it is technically challenging and its long-term results are unclear.

The technical difficulty of CABG in infants mainly comes from the small caliber of the graft. In a study of patients with Kawasaki disease, the 15-year patency rate of IMA grafts was $91 \%$ in patients aged $>12$ years compared with $65 \%$ in patients aged $<12$ years. This is mainly attributable to the diameter of the artery being too small for anastomosis [2]. For improved outcomes, skilled surgical hands are essential. However, researchers have argued that anastomosis between the internal mammary and coronary arteries is feasible in infants because their coronary arteries are usually $\geq 1 \mathrm{~mm}$ in diameter. The lower limit for anastomosis is suggested to be $0.7 \mathrm{~mm}$ in modern microsuture techniques [3]. In our case, preoperative aortography revealed that the diameter of the LIMA was $1 \mathrm{~mm}$.

In order to reduce ischemic injury to the myocardium, the on-pump beating heart technique was used. In this patient, coronary os insufficiency raised concerns regarding the possibility of inadequate delivery of the cardioplegia solution during aortic cross-clamping. By maintaining the heart beating without cross-clamping, the on-pump beating technique has benefits for myocardial protection and unloading of the heart. It was possible to stabilize the beating heart by using the Octopus tissue stabilizer (Medtronic Inc., Minneapolis, MN, USA). The IMA is preferred to a saphenous vein graft in patients with congenital heart disease because it enables better patency. One concern is that the IMA graft provides insufficient perfusion of the growing heart. However, a case of a patient who survived until adulthood after undergoing bilateral IMA grafting for complete obstruction of both coronary origins has been reported [4]. In this regard, the IMA graft is a reasonable choice for CABG in infants. On preoperative coronary angiography, the LCx also showed diminished flow. However, it is thought that the proximal portion of the left main coronary artery was the main lesion, which means that the LCx can be perfused by reverse flow from the revascularized LAD flow.

Percutaneous transluminal coronary artery angioplasty (PTCA) can also be an alternative treatment for post-ASO coronary stenosis. A study reported 5-year follow-up data of 7 cases of coronary stenosis after ASO who underwent PTCA [5]. In that study, all the stenoses were successfully treated with PTCA, and follow-up angiography at a mean interval of 50 months revealed normal growth of the coronary arteries. The age of the youngest patient was 3 weeks. However, percutaneous intervention was not considered in our case, as our center had limited experience with percutaneous intervention and the coronary anatomy of the patient become complicated after the revision.

Although its long-term results are not well established, some cases of IMA grafting for post-ASO coronary complications have been reported. IMA grafting in a neonate was first described in 1997 by Yaku et al. [6]. Ever since, acceptable outcomes of post-ASO CABG have been reported. In a case series reported by Legendre et al. [7] in 2010, 12 patients underwent ASO-related CABG. After a mean follow-up of 54 months, most of the grafts were patent, 1 patient died from coronary occlusion, and 2 patients showed stenotic lesions. A recent multicenter study of pediatric $C A B G$ in Europe reported 65 cases of pediatric CABG (33 patients aged $<12$ months), excluding coronary complications of Kawasaki disease [8]. In the study, 11 in-hospital and 3 late deaths were reported. The mammary grafts used in ASO remain patent for approximately 12 years postoperatively. Frequent and lifelong follow-up is necessary, as these patients easily develop coronary restenosis and ischemic cardiomyopathy.

In conclusion, $\mathrm{CABG}$ can be an alternative option for post-ASO coronary complications in infants. However, its long-term outcomes should be studied further.

\section{Conflict of interest}

No potential conflict of interest relevant to this article was reported.

\section{ORCID}

Wooseok Choi: https://orcid.org/0000-0002-7073-0442 Wonkyung Pyo: https://orcid.org/0000-0002-5769-052X Eun Seok Choi: https://orcid.org/0000-0002-0618-4686 Cheol Hyun Chung: https://orcid.org/0000-0001-8981-6011 


\section{References}

1. Bonnet D, Bonhoeffer P, Sidi D, et al. Surgical angioplasty of the main coronary arteries in children. J Thorac Cardiovasc Surg 1999; 117:352-7.

2. Kitamura S, Kameda Y, Seki T, et al. Long-term outcome of myocardial revascularization in patients with Kawasaki coronary artery disease: a multicenter cooperative study. J Thorac Cardiovasc Surg 1994;107:663-73.

3. Mavroudis C, Backer CL, Muster AJ, et al. Expanding indications for pediatric coronary artery bypass. J Thorac Cardiovasc Surg 1996; 111:181-9.

4. Nair KK, Chan KC, Hickey MS. Arterial switch operation: successful bilateral internal thoracic artery grafting. Ann Thorac Surg 2000;
69:949-51.

5. Kampmann C, Kuroczynski W, Trubel H, Knuf M, Schneider M, Heinemann MK. Late results after PTCA for coronary stenosis after the arterial switch procedure for transposition of the great arteries. Ann Thorac Surg 2005;80:1641-6.

6. Yaku H, Nunn GR, Sholler GF. Internal mammary artery grafting in a neonate for coronary hypoperfusion after arterial switch. Ann Thorac Surg 1997;64:543-4.

7. Legendre A, Chantepie A, Belli E, et al. Outcome of coronary artery bypass grafting performed in young children. J Thorac Cardiovasc Surg 2010;139:349-53.

8. Vida VL, Torregrossa G, De Franceschi M, et al. Pediatric coronary artery revascularization: a European multicenter study. Ann Thorac Surg 2013;96:898-903. 